\title{
Performance of the LACE index to predict 30-day hospital readmissions in patients with chronic obstructive pulmonary disease
}

This article was published in the following Dove Press journal:

Clinical Epidemiology

\author{
Maryam A Hakim ${ }^{1,2}$ \\ Frances L Garden ${ }^{2,3}$ \\ Matthew D Jennings ${ }^{4}$ \\ Claudia C Dobler $1,2,3,5$ \\ 'Department of Respiratory Medicine, \\ Liverpool Hospital, ${ }^{2}$ South Western \\ Sydney Clinical School, University of \\ New South Wales, ${ }^{3}$ Ingham Institute \\ for Applied Medical Research, \\ ${ }^{4}$ Department of Physiotherapy, \\ Liverpool Hospital, Sydney, NSW, \\ Australia; ${ }^{5}$ Evidence-Based Practice \\ Center, Robert D. and Patricia E. Kern \\ Center for the Science of Health \\ Care Delivery, Mayo Clinic, Rochester, \\ MN, USA
}

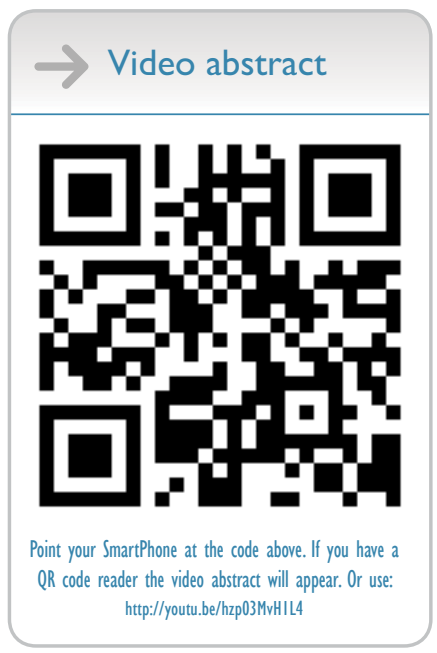

Correspondence: Claudia C Dobler Department of Respiratory Medicine, Liverpool Hospital, Elizabeth Street, Liverpool, NSW 2034, Australia

Tel +6I 0298283000

Email c.dobler@unsw.edu.au
Background and objective: Patients hospitalized for acute exacerbation of chronic obstructive pulmonary disease (COPD) have a high 30-day hospital readmission rate, which has a large impact on the health care system and patients' quality of life. The use of a prediction model to quantify a patient's risk of readmission may assist in directing interventions to patients who will benefit most. The objective of this study was to calculate the rate of 30-day readmissions and evaluate the accuracy of the LACE index (length of stay, acuity of admission, co-morbidities, and emergency department visits within the last 6 months) for 30-day readmissions in a general hospital population of COPD patients.

Methods: All patients admitted with a principal diagnosis of COPD to Liverpool Hospital, a tertiary hospital in Sydney, Australia, between 2006 and 2016 were included in the study. A LACE index score was calculated for each patient and assessed using receiver operator characteristic curves.

Results: During the study period, 2,662 patients had 5,979 hospitalizations for COPD. Four percent of patients died in hospital and 25\% were readmitted within 30 days; $56 \%$ of all 30-day readmissions were again due to COPD. The most common reasons for readmission, following COPD, were heart failure, pneumonia, and chest pain. The LACE index had moderate discriminative ability to predict 30-day readmission $(C$-statistic $=0.63)$.

Conclusion: The 30-day hospital readmission rate was $25 \%$ following hospitalization for COPD in an Australian tertiary hospital and as such comparable to international published rates. The LACE index only had moderate discriminative ability to predict 30 -day readmission in patients hospitalized for COPD.

Keywords: predictor model, risk prediction, unwarranted variation

\section{Introduction}

Many patients with chronic conditions are frequent users of the health care system and need to be admitted to hospital repeatedly, often within short intervals. Hospital readmissions, frequently measured as 30-day readmissions (occurring within 30 days of discharge), have a significant impact on patients' quality of life, ${ }^{1}$ add to the disease and treatment burden experienced by COPD patients, ${ }^{2,3}$ are a burden on the health care system, and put a strain on hospital resources. ${ }^{4}$ In $2003-2004,19.6 \%$ of the $11,855,702$ Medicare beneficiaries in the USA who had been discharged from a hospital were rehospitalized within 30 days. ${ }^{5}$

The number of 30-day hospital readmissions has been steadily rising in high income countries over the last decade. In Scotland, readmission rates went from 20.5 to $24.5 \%$ from 1996 to $2005 .{ }^{6}$ In England, there was a $27 \%$ increase from 2000 to $2012 .^{7}$ The 
reasons for this are not entirely clear but may potentially be associated with the increasing prevalence of chronic diseases as well as changes in the organization and delivery of health care with shorter lengths of stay in hospital and efforts to increasingly care for patients in an outpatient setting. In some countries, such as the USA and Australia, financial penalties for hospitals have been implemented to reduce readmission rates. In the USA, readmission reduction programs are implemented with an added penalty to hospitals if they have excess readmission rates among their Medicare patients; in 2015, $\$ 280$ million dollars in Medicare funds were withheld from 2,217 hospitals. ${ }^{8,9}$ In the state of New South Wales, Australia (the setting of this study), hospitals with readmission rates above the state average receive decreased funding in their annual budgets. In 2015 and 2016, underperforming hospitals were penalized millions of dollars.

Chronic obstructive pulmonary disease (COPD) has one of the highest rates of 30-day hospital readmissions, along with congestive heart failure and pneumonia. ${ }^{10}$ COPD readmission rates vary across countries and hospitals. In 2014, a quarter of all states in the USA had high 30-day hospital readmission rates ranging from 23 to $28 \%,{ }^{11}$ and among Medicare beneficiaries, the national average readmission rate was $19.6 \%{ }^{5}$ In New South Wales, Australia, there were 22 readmissions per 100 hospitalizations for COPD (total of 47,359 index hospitalizations) within 30 days in 2012-2015, of whom $55 \%$ were again for a principal diagnosis of COPD and $10 \%$ were for a condition related to COPD. ${ }^{12}$

There is a multitude of predictor models that aim to predict individual patient's risk of short-term hospital readmission, with a 2016 systematic review identifying 73 unique models. ${ }^{13}$ Models predicted either 28-day or 30-day readmissions for all causes or specifically for medical, surgical, or mental health diagnoses. An optimal readmission risk prediction model strikes a good balance between discriminative ability and model simplicity. One model that meets this criterion is the LACE index (length of stay, acuity of admission, co-morbidities, emergency department visits within the last 6 months). ${ }^{14}$ The LACE index was discriminative with the $C$-statistic (area under the receiver operator characteristic [ROC] curve) of 0.684 in predicting readmissions and mortality within 30 days in a general hospital population it was originally tested in. ${ }^{14}$ In addition, it is relatively simple to use in clinical care compared to other predictor models as it uses patient characteristics that can be easily obtained by health care professionals. The model was originally tested on a mixed cohort of hospital patients consisting of elective and nonelective, medical, and surgical patients. Its predictive ability has been tested for some specific diseases (eg, heart failure), ${ }^{15}$ but little is known about its ability to predict the risk of short-term hospital readmission in patients with COPD. A relatively small study applied the LACE index to 461 COPD patients in the USA and did not find that the LACE index predicted 30-day all-cause hospital readmissions. ${ }^{16}$ The aim of this study was to evaluate the ability of the LACE index to predict 30-day all-cause hospital readmissions in a large cohort of patients with COPD in Australia.

\section{Methods}

\section{Study design and setting}

This was a retrospective cohort study from January 2006 to January 2016, using data extracted from the electronic hospital management system of Liverpool Hospital, a tertiary hospital in Sydney, Australia. The hospital is located in an area of low socioeconomic status with many migrants of non-English speaking background. The study cohort comprised patients older than 18 years with a hospitalization for COPD as principal diagnosis, identified through International Classification of Diseases-10 codes (J44.0, J44.1, J44.8, and J44.9). Patients discharged to a nursing home were excluded. Ethical approval was obtained from the South Western Sydney Local Health District Human Research Ethics Committee (HREC/15/LPOOL/557). The requirement to obtain patient consent was waived because this was a retrospective database study. Patient confidentiality was maintained by de-identification of all data and aggregation of potentially identifiable variables to a level that precludes identification.

\section{Data analysis}

A 30-day readmission was defined as a readmission for any diagnosis following an index hospitalization with a principal diagnosis of COPD. The LACE index (length of stay, acuity of admission, comorbidities [based on the Charlson comorbidity index $],{ }^{17}$ and emergency visits within the last 6 months) was used to calculate a score for each patient. All patients were given the maximum score of 3 points for acuity because all patients with COPD were admitted via the emergency department. The predictive ability of the LACE index in the study cohort was assessed using ROC curves. The outcomes tested were readmission within 30 days and death during index hospitalization combined and 30-day readmission alone. ROC curves were obtained to assess the sensitivity and specificity of the predictor model. The association between each of the LACE variables and readmission was assessed using a generalized estimating equation. To assess if the predictive ability of the LACE model could be improved, the 
following five different LACE models were assessed, each modified by substituting the 3 points for acuity of admission for: presence of mental health disorders (F01-F99), presence of heart failure (I50.00-I50.43), admission during a weekend, admission after core hours, and a male patient.

A Chi-square of trend was used to assess if readmission rates increased over time. A generalized estimating equation was used to test if there was a significant interaction term between readmission rates and length of stay over time.

Statistical analysis was conducted using SAS 9.4 (SAS Institute Inc., Cary, NC, USA).

\section{Results}

The study cohort included 2,662 patients. They accounted for 26,789 hospitalizations during the study period, of which 5,979 hospitalizations were for COPD. More than half of the patients were male $(53.3 \%)$ with an average age of 72.5 ( $\mathrm{SD}=12$, range: $21-99$ ) years. All patients had a minimum Charlson score of 1 by definition (attributed to COPD).

Of 5,979 index hospitalizations for COPD, 25.1\% resulted in a hospital readmission within 30 days, of which $56.0 \%$ were due to COPD. The other main reasons for readmission during 30 days and 1 year were heart failure and pneumonia (Table 1). On average, patients were readmitted within 13.3 days, with 7 days being the most common time for readmission. Readmission rates for any diagnosis within 90,180 , and 366 days were $43.5,54.8$, and $65.2 \%$, respectively. Readmission rates for COPD only within 30, 90, 180, and 366 days were $14.2,23.9,29.4$, and $34.0 \%$, respectively. During index hospitalizations for COPD, 239 (4.0\%) patients died in hospital.

Readmission rates per year over the last decade gradually increased from $22.6 \%$ in 2006 to $27.9 \%$ in 2015 (Figure 1).

Table I Reasons for readmission within 30 days and 366 days following an index hospitalization for COPD

\begin{tabular}{lll}
\hline Diagnosis & \multicolumn{2}{l}{ Number of readmissions } \\
\cline { 2 - 3 } & $\begin{array}{l}\text { Within 30 days, } \\
\text { n (\%) }\end{array}$ & $\begin{array}{l}\text { Within 366 days, } \\
\text { n (\%) }\end{array}$ \\
\hline COPD & $847(56)$ & $2,034(52)$ \\
Heart failure & $39(2.7)$ & $117(3)$ \\
Pneumonia & $29(1.9)$ & $77(2)$ \\
Chest pain & $21(1.4)$ & $78(2)$ \\
Myocardial infarction & $17(1)$ & $40(1)$ \\
Cellulitis & $15(1)$ & $32(<1)$ \\
Asthma & $11(<1)$ & $42(1)$ \\
Others & $540(36)$ & $1,593(38)$ \\
\hline
\end{tabular}

Abbreviation: COPD, chronic obstructive pulmonary disease.
The average length of stay for patients readmitted within 30 days decreased from 9.1 days in 2006 to 5.7 days in 2015 (Figure 2). There was a significant trend in increasing readmission rates over time $(P=0.007)$; however, increased readmission rates and decreased length of stay were not statistically significantly associated $(P=0.93)$.

The average LACE score for each index admission of COPD was 9.8 points (out of a possible range of 1-19 for patients with COPD); the distribution of scores is shown in Figure 3 . The odds ratios of the association between the different LACE variables and the risk of 30-day readmission (for a one category increase within each variable) were 1.02 (95\% CI 0.94-1.10) for the length of stay, 1.00 (95\% CI 0.93-1.09) for comorbidity, and 1.25 (95\% CI 1.21-1.29) for the number of emergency visits in the last 6 months. The acuity criterion from the LACE index was not relevant to our cohort as every patient was admitted through the emergency room, and there were no elective admissions.

The ROC analysis, using the outcome of 30-day readmissions, showed an area under the curve (Figure 4 ) or $C$-statistic of 0.63 (95\% CI 0.62-0.65). When including the outcomes death or 30-day readmissions, the $C$-statistic was also 0.63 (95\% CI 0.62-0.65). This demonstrated that the LACE model has only moderate predictive ability for 30 -day readmission following hospitalizations for COPD. The sensitivity and specificity of the model varied based on the cutoff scores between high and low risk patients for 30-day readmission (Table 2). Figure 5 illustrates the percentage of 30-day readmissions per given LACE score.

When using a cutoff point of LACE score $\geq 10$ (a cutoff previously used by another study and found to have a good discriminatory ability to predict the risk of 30-day readmission ${ }^{18}$ ), $48.5 \%$ of the cohort were in the high-risk group. In this high-risk group, $32.8 \%$ of patients were readmitted within 30 days (ie, positive predictive value of the model). In the low-risk group, $17.9 \%$ of patients were readmitted within 30 days and $82.1 \%$ were not readmitted (ie, negative predictive value of the model). The sensitivity of using a cutoff point of $\geq 10$ was 0.63 , and the specificity was 0.56 . Overall, of the 1,502 readmissions $(25.1 \%$ of all COPD index hospitalizations), $63.4 \%$ of readmissions had a LACE score of $\geq 10$.

Modification of the LACE index by replacing the acuity variable, as it was not relevant to our cohort, showed no improvement in the predictive ability of the LACE index. The $C$-statistic from each model was as follows: mental health disorders $=0.626$ (95\% CI $0.61-0.64)$, heart failure 


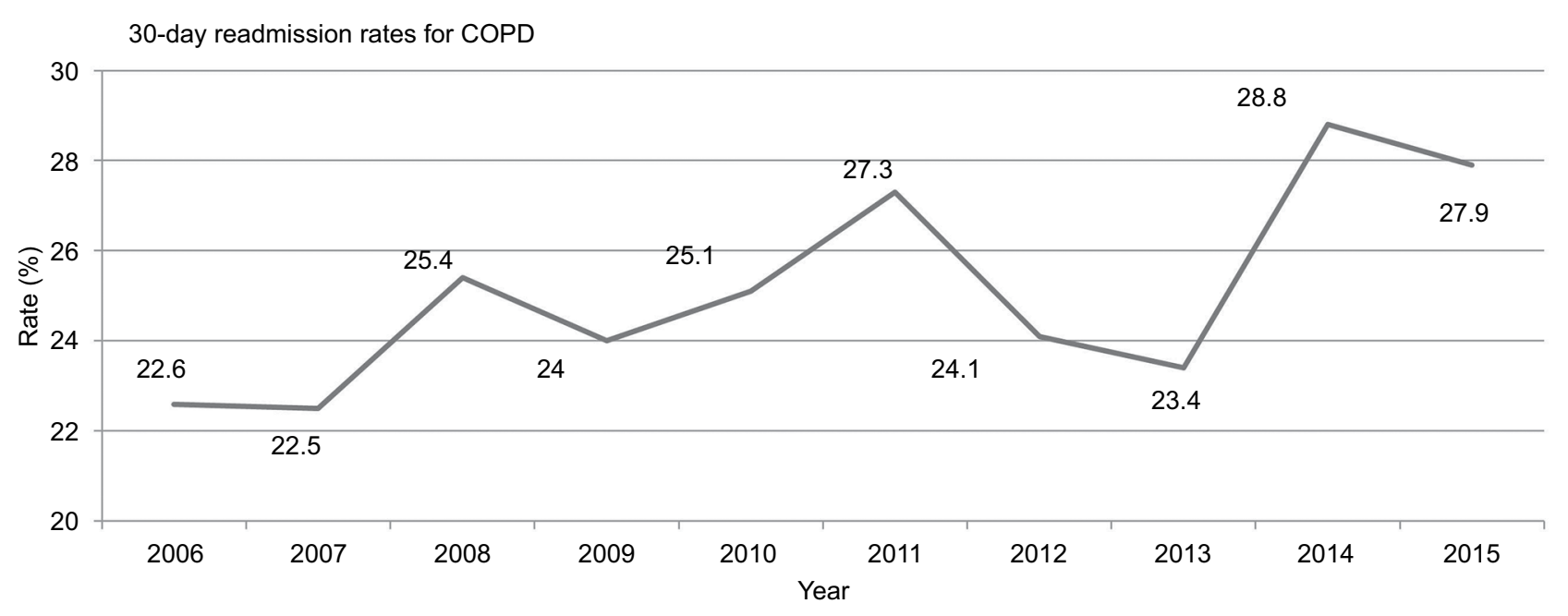

Figure I Thirty-day readmission rates from 2006 to 2015 for any diagnosis following an index hospitalization of COPD. Abbreviation: COPD, chronic obstructive pulmonary disease.

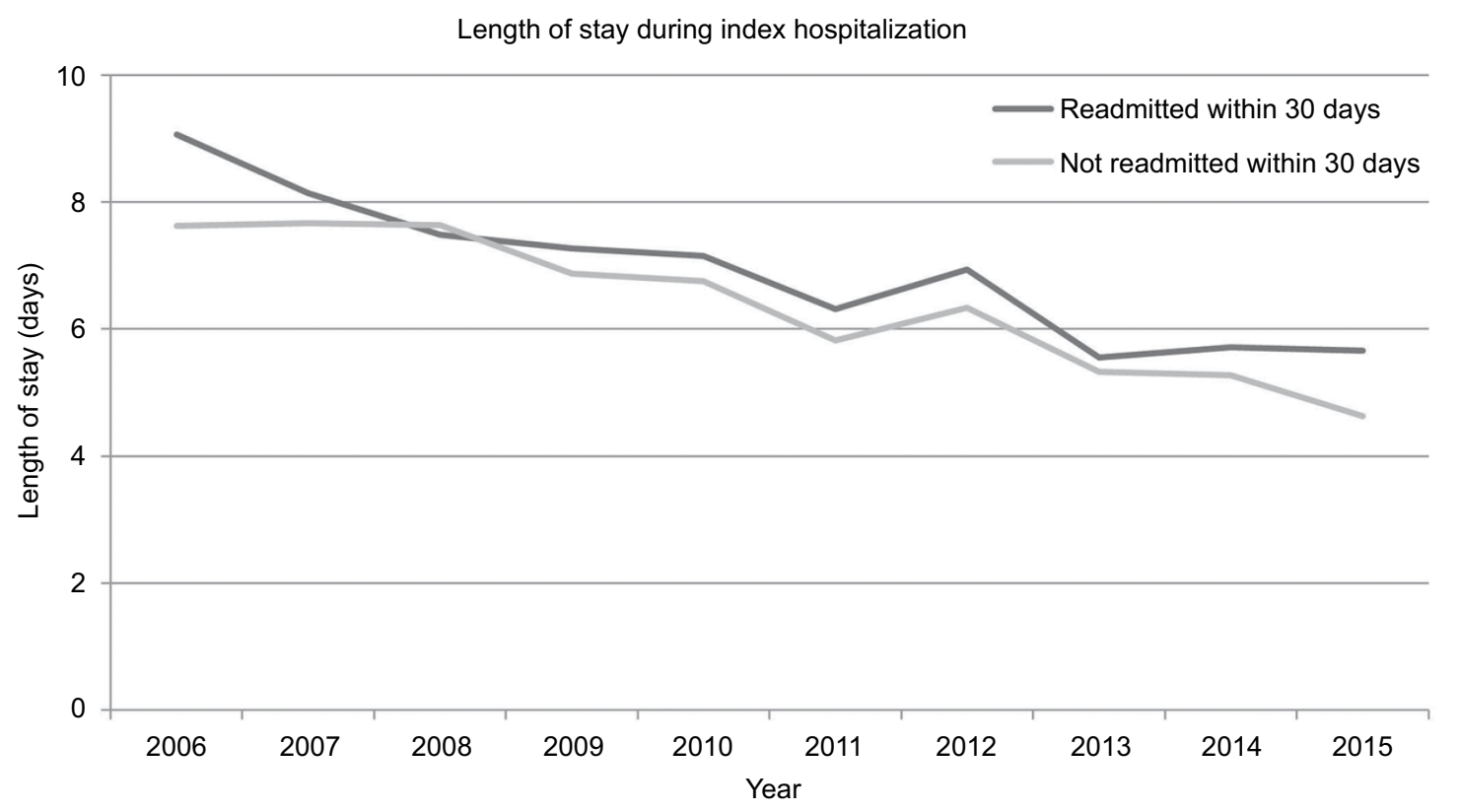

Figure 2 Length of stay during index hospitalization for COPD from 2006 to 2015 in patients who were readmitted within 30 days and those who were not readmitted. Abbreviation: COPD, chronic obstructive pulmonary disease.

$=0.629(95 \%$ CI $0.61-0.65)$, admission during a weekend $=0.618(95 \%$ CI $0.60-0.63)$, admission after core hours $=0.617(95 \%$ CI $0.60-0.63)$, and being male $=0.6305(95 \%$ CI $0.61-0.65)$.

\section{Discussion}

In our cohort, one in four patients admitted to hospital for COPD was readmitted within 30 days. More than half of these patients were readmitted for COPD exacerbations, followed by other respiratory and cardiovascular diagnoses.
The LACE index was only moderately predictive for 30-day hospital readmissions, possibly due to its simplicity.

A systematic review identified 73 unique predictor models for 28 or 30 days unplanned hospital readmissions in both broader and specific patient populations. ${ }^{13}$ The predictive ability of these models vastly varied with $C$-statistic between 0.21 and 0.88 , with the better performing models generally having more variables. In clinical practice, it is important that the model is simple and contains variables that can be easily obtained; however, this means that the predictive ability of 


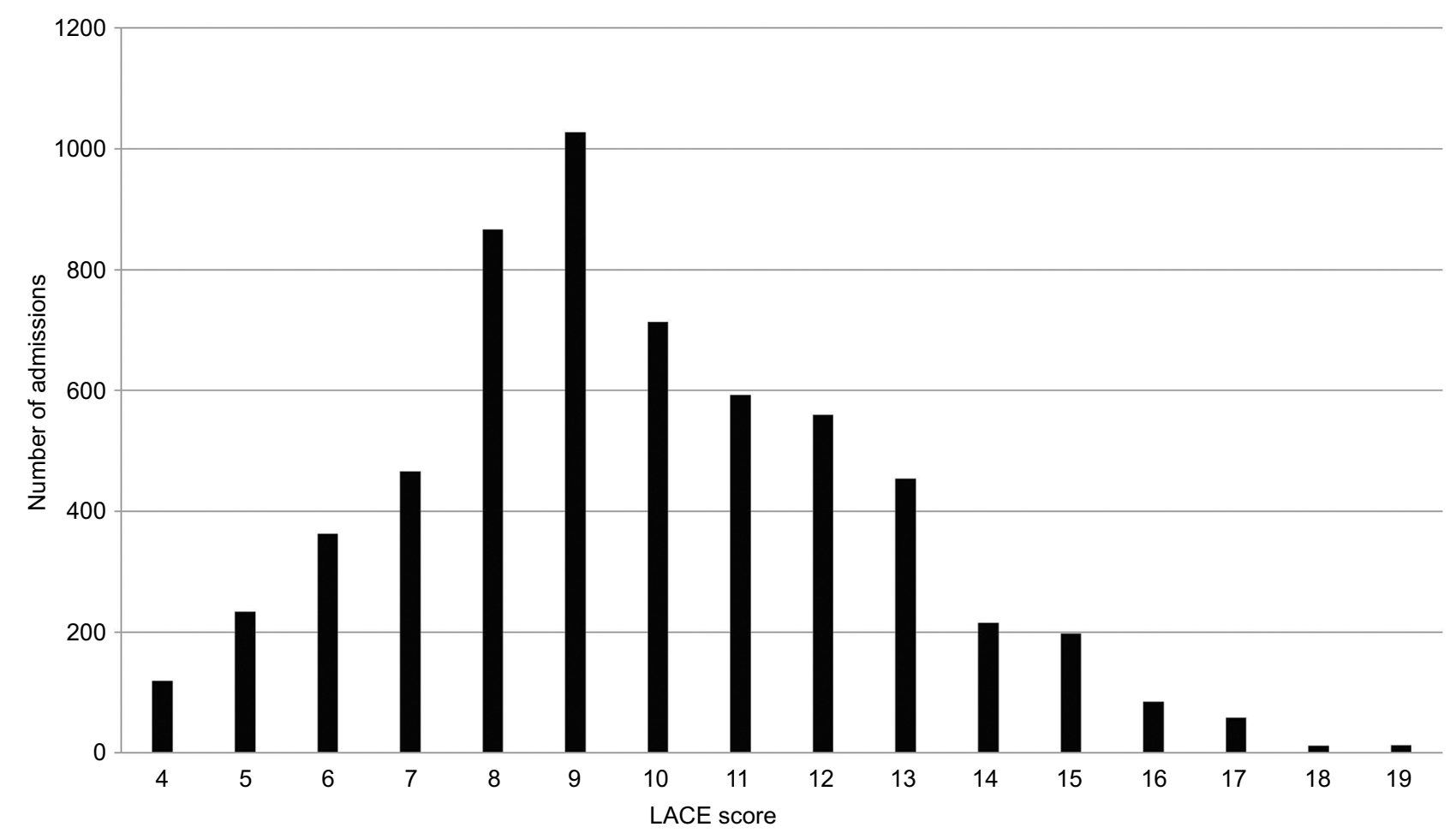

Figure 3 Distribution of LACE scores among the COPD cohort. Abbreviation: COPD, chronic obstructive pulmonary disease.

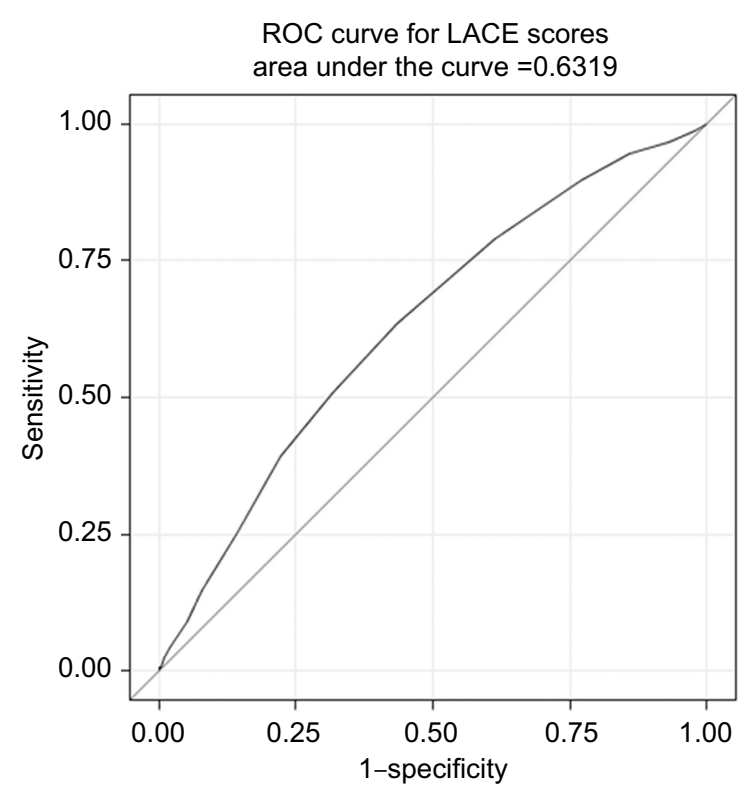

Figure 4 ROC curve for the LACE index in COPD.

Notes: The ROC curve illustrates the performance of the LACE index for predicting 30-day readmission at different cutoff points. With increased sensitivity, specificity is decreased and vice versa. The area under the curve, which is equal to the $C$-statistic, is 0.63 , indicating a moderate predictive ability of the model for identifying patients at risk of readmission within 30 days.

Abbreviations: COPD, chronic obstructive pulmonary disease; ROC, receiver operator characteristic.

the model may be compromised. A 2012 study on predictors of readmission in patients with COPD found six variables (at least two COPD exacerbations within 1 year, body mass index, number of previous surgeries, type of antibiotics
Table 2 Sensitivity and specificity for each cutoff point of the LACE index in COPD patients

\begin{tabular}{lll}
\hline LACE score & Sensitivity & Specificity \\
\hline 4 & 1.00 & 0.00 \\
5 & 0.99 & 0.02 \\
6 & 0.97 & 0.07 \\
7 & 0.94 & 0.14 \\
8 & 0.90 & 0.23 \\
9 & 0.79 & 0.39 \\
10 & 0.63 & 0.56 \\
11 & 0.51 & 0.68 \\
12 & 0.39 & 0.78 \\
13 & 0.25 & 0.85 \\
14 & 0.15 & 0.92 \\
15 & 0.09 & 0.95 \\
16 & 0.04 & 0.98 \\
17 & 0.02 & 0.99 \\
18 & 0.01 & 1.00 \\
19 & 0.01 & 1.00 \\
\hline
\end{tabular}

Note: Each score identifies individuals in the high-risk group for hospital readmission, for example, at 10 , admissions with a score of $\geq 10$ are identified as returning to hospital within 30 days by the LACE index.

Abbreviation: COPD, chronic obstructive pulmonary disease.

administered, and albumin level) that were correlated with 30-day readmissions and a $C$-statistic of $0.72 .{ }^{19}$ Another study focusing on readmissions in COPD patients found nine variables, such as being male, history of osteoporosis, and no statin prescription, to be associated with increased risk of readmission $(C$-statistic $=0.68) .{ }^{20}$ However, neither 


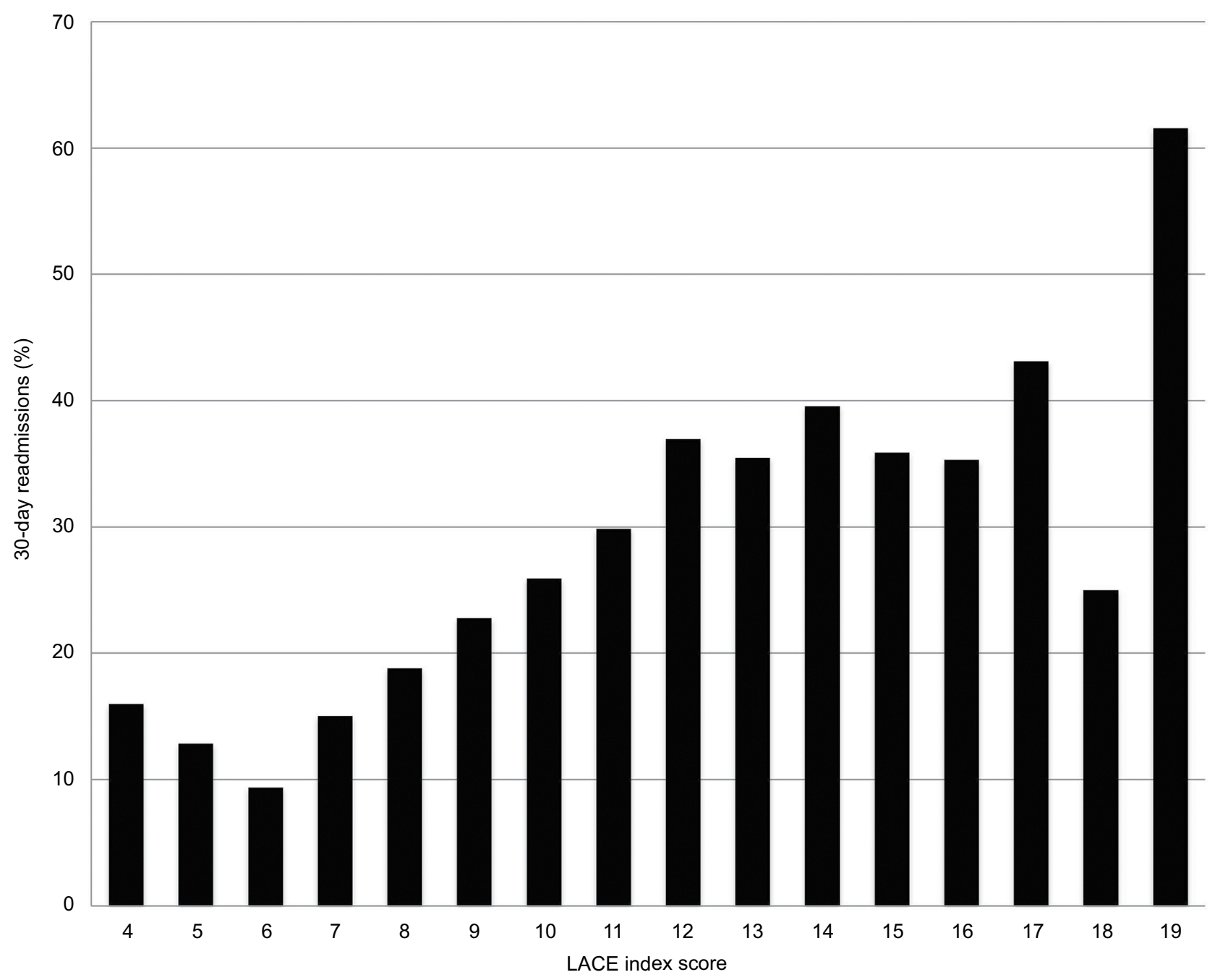

Figure 5 Correlation between 30-day readmissions and LACE index score.

study integrated the variables into a scoring model that can be clinically used without the assistance of a statistical analysis program.

The LACE index has been previously tested in different populations with mixed results. The original study with a mixed hospital population had a $C$-statistic of 0.68 . In a cohort of heart failure patients, the $C$-statistic was 0.56 , indicating that it performed only slightly better than chance. ${ }^{15}$ In a cohort of all patients discharged from hospital with a mean age of 84.8 years, the LACE also performed poorly. The median LACE score between those readmitted and those who were not was 12.5 vs $12(P=0.13)$ with a $C$-statistic of $0.60 .{ }^{21}$ On the other hand, another study found the LACE index to be a good predictor for readmission in their cohort of all patients discharged from six hospitals in Toronto, Canada. They found that the high-risk group was twice more likely to be readmitted within 30 days than the low-risk group (relative risk 2.1 95\% CI: $1.9-2.2)^{22}$
The PEARL study developed a new prediction model for 90-day hospital readmission for patients with COPD. The criteria they used (PEARL) included previous admissions, extended Medical Research Council dyspnoea scale (eMRCD), age, right-sided heart failure, and left-sided heart failure. Their $C$-statistic for predicting which patients would be readmitted within 90 days was $0.73 .{ }^{23}$ In the same study, other prediction tools were tested, including the LACE index, which had a $C$-statistic of 0.65 for predicting 90 -day readmissions.

A relatively small study conducted in 2013 in the USA applied the LACE index to 461 COPD patients and found that a LACE score of $\geq 13$ was not associated with 30-day readmissions $(P=0.186) .{ }^{16}$ Importantly, because of big differences in health care systems globally, the predictive validity of the LACE index (or any other index) needs to be tested in the setting where it is going to be applied.

Generic prediction models, such as the LACE index, tested on COPD and other patient populations have 
highlighted the heterogeneity in patient populations. Many predictors can be very specific for a certain patient population but are not relevant to another. Acuity of the admission, for example, which is included in the LACE index, is almost completely irrelevant in COPD, as almost all hospitalizations for COPD are due to exacerbations of COPD and are thus acute and not elective. Having a COPD-specific prediction tool can ensure that all the important factors that increase patients' risk of readmission can be included to maximize the predictive ability of the model.

Strengths of our study are the long observation period (10 years) and the relatively large cohort of patients $(n=2,662)$. The 10-year time period allowed us to look at trends in readmission rates and length of stay and how they have changed over time. A limitation of our study was that information was only available on readmissions to the same hospital where the patients had been hospitalized for their index hospitalization. In New South Wales, Australia, 15\% of COPD patients represented to a different hospital than their index hospital. ${ }^{12}$ Not capturing 30-day readmissions of patients who had an index admission at the study hospital but presented to another hospital for readmission would have resulted in underestimation of the readmission risk. In contrast, there would have been patients who had an index hospitalization at a different hospital and presented to the study hospital for readmission and they would not have been included in our analysis as readmissions. Therefore, in the end, there is likely minimal directional bias as index hospitalizations and rehospitalizations would have been missed.

Epidemiological studies have shown that up to $20.9 \%$ of COPD patients have concurrent heart failure. ${ }^{24}$ Each disease may have an impact on the disease progression of the other; however, the effects of interactions between these diseases on patients and hospital readmission rates are unclear. ${ }^{25}$ In our cohort, $13.4 \%$ of COPD patients had heart failure; however, it did not increase their risk of 30-day readmission (odds ratio [OR]: 1.22 95\% CI: 0.80-1.86).

A prospective cohort study in the UK found that depressive symptoms predict risk of 30-day readmission in COPD patients (OR: 1.30, 95\% CI: 1.06-1.60, $P=0.013) .{ }^{26}$ While there was a high rate of mental health disorders $(22.3 \%)$ among our COPD cohort, it was not significantly correlated with 30-day readmissions (OR: 1.19, 95\% CI: 0.88-1.62).

In our study, there was a trend showing that during the 10-year study period, as length of stay decreased, 30-day readmissions increased. While some studies have shown that there is a correlation between the two variables, others have found none. ${ }^{27}$ In our cohort, the association between length and stay and readmission risk was not statistically significant. The clinical relevance of the trend remains unclear.

High 30-day readmission rates can potentially be the result of insufficient patient management practices and postdischarge care; however, many chronic diseases will lead to repeat hospital admissions despite best possible care. Studies have shown that COPD exacerbations occur in clusters. ${ }^{28}$ Therefore, 30-day readmissions for COPD exacerbation are common and often inevitable despite optimal management. In our study, $56.0 \%$ of readmissions (after index hospitalization for COPD) were again due to COPD, which was significantly higher than in a large study from the USA, in which COPD was only responsible for $27.6 \%$ of all readmissions. ${ }^{29}$ Whether this indicates a higher proportion of unavoidable readmissions in our cohort is, however, unclear. Discrepancies in readmission rates between different settings and over time suggest that at least some readmissions are potentially avoidable and can be reduced with appropriate interventions. ${ }^{4}$ Unavoidable readmissions may occur due to the natural progression of an underlying disease or a diagnosis unrelated to the index admission. ${ }^{30}$ The proportion of readmissions that are preventable is unclear. A systematic review of 34 studies found the range of avoidable readmission between 5 and $79 \%$, with a median of $27 \%$ of all 30 -day readmissions. ${ }^{31}$

Possible interventions that may be utilized to reduce the number of preventable readmissions have been tested in various studies. A postdischarge care bundle plan applied to a cohort of 44 COPD patients found the intervention group to have a 30 -day readmission rate of $9.1 \%$ compared to $54.5 \%$ in the control group. ${ }^{32}$ Two other studies also demonstrated a decrease in 30-day readmissions when utilizing care bundles, $14.7 \%$ from $22.7 \%{ }^{33}$ and $15.4 \%$ from $17.4 \%{ }^{34}$ However, not all interventions work. A randomized controlled trial using a new comprehensive care management program was terminated after 1 year due to increased deaths in the intervention group than in the control. The discrepancy in the effectiveness of different interventions highlights the importance of continuing research in this field to not only reduce readmissions in COPD patients but also improve patient-important outcomes.

It is important to note that the use of risk assessment tools to predict hospital readmissions emerged in the context of health services/administrations aiming to ensure service efficiency and reduce readmissions. The role of predicting patients' risk for hospital readmission in a clinical context is less clear. It has been argued that identification of patients at high risk of 30-day readmission allows care processes and eventually patient-important outcomes to be improved, but 
the evidence to support this assumption is currently insufficient and the relationship between the process measure "30day hospital readmission" and patient-important outcomes such as mortality and health-related quality of life is poorly understood.

No doubt, hospitalizations, and possibly even more so, hospital readmissions, interfere with a patient's daily living and will (at least temporarily) reduce a patient's health-related quality of life. Whether reducing hospital readmissions will go hand-in-hand with improving patient-important outcomes is, however, unclear and will likely depend on the intervention used to reduce readmissions. When frail patients are acutely unwell, misguided efforts to keep them out of the hospital might increase their risk of an adverse outcome by delaying necessary acute care. A study published in 2016 that analyzed data from the US Centers for Medicare and Medicaid from hospitals across the USA found that hospitals with the highest rates of readmission were actually more likely to show better mortality scores in patients treated for heart failure, COPD, and stroke. ${ }^{35}$ These data certainly caution us against overemphasizing the importance of 30-day hospital readmissions as a measure for the quality of patient care.

\section{Conclusion}

Predictor models for the risk of 30-day hospital readmission can potentially be useful to define a target population who will most likely benefit from a tailored discharge and/or follow-up intervention to reduce the risk of readmission. In the absence of a simple yet highly accurate prediction tool for readmissions in patients with COPD, the LACE index can serve as user-friendly tool with moderate (but better than chance) predictive ability, especially in a context where sparse resources need to be focused on patients with the biggest potential need. Increasing the LACE score threshold will increase the positive predictive value of the tool; however, more patients who will be readmitted would be missed.

Future research should focus on assessing the association between hospital readmissions and patient-important outcomes; in particular, it should examine the effect of interventions to reduce hospital readmissions in patients with COPD on patient-important outcomes, such as health-related quality of life and mortality.

\section{Acknowledgment}

CCD was supported by an Australian National Health and Medical Research Council fellowship (APP1123733) and an award from the South Western Sydney Local Health District.

\section{Disclosure}

The authors report no conflicts of interest in this work.

\section{References}

1. Osman IM, Godden DJ, Friend JA, Legge JS, Douglas JG. Quality of life and hospital re-admission in patients with chronic obstructive pulmonary disease. Thorax. 1997;52(1):67-71.

2. Harb N, Foster JM, Dobler CC. Patient-perceived treatment burden of chronic obstructive pulmonary disease. Int J Chron Obstruct Pulmon Dis. 2017;12:1641-1652.

3. Dobler CC. Defragmenting health care for patients with chronic obstructive pulmonary disease. AMJ. 2016;9(6):185-188.

4. Hansen LO, Young RS, Hinami K, Leung A, Williams MV. Interventions to reduce 30-day rehospitalization: a systematic review. Ann Intern Med. 2011;155(8):520-528.

5. Jencks SF, Williams MV, Coleman EA. Rehospitalizations among patients in the Medicare fee-for-service program. $N$ Engl $J$ Med. 2009;360(14):1418-1428.

6. Audit Scotland. Managing Long-term Conditions. 2007. Available from: http://www.audit-scotland.gov.uk/docs/health/2007/nr_070816_managing_long_term.pdf. Accessed November 2, 2017.

7. Harries TH, Thornton H, Crichton S, Schofield P, Gilkes A, White PT. Hospital readmissions for COPD: a retrospective longitudinal study. NPJ Prim Care Respir Med. 2017;27:31.

8. Feemster LC, Au DH. Penalizing hospitals for chronic obstructive pulmonary disease readmissions. Am J Respir Crit Care Med. 2014;189(6):634-639.

9. Joynt KE, Jha AK. A path forward on Medicare readmissions. $N$ Engl J Med. 2013;368(13):1175-1177.

10. Shams I, Ajorlou S, Yang K. A predictive analytics approach to reducing 30-day avoidable readmissions among patients with heart failure, acute myocardial infarction, pneumonia, or COPD. Health Care Manag Sci. 2015;18(1):19-34.

11. Sjoding MW, Cooke CR. Readmission penalties for chronic obstructive pulmonary disease will further stress hospitals caring for vulnerable patient populations. Am J Respir Crit Care Med. 2014;190(9): 1072-1074.

12. Bureau of Health Information [webpage on the Internet]. The Insights Series - Exploring Clinical Variation in Readmission, Return to Acute Care Following Discharge from Hospital, Eight Clinical Conditions, NSW, July 2012 - June 2015. Sydney, NSW: BHI; 2017. Available from: http://www.bhi.nsw.gov.au/BHI_reports/Insights_Series/ clinical-variation-in-readmission/_nocache. Accessed November 2, 2017.

13. Zhou H, Della PR, Roberts P, Goh L, Dhaliwal SS. Utility of models to predict 28-day or 30-day unplanned hospital readmissions: an updated systematic review. BMJ Open. 2016;6(6):e011060.

14. van Walraven C, Dhalla IA, Bell C, et al. Derivation and validation of an index to predict early death or unplanned readmission after discharge from hospital to the community. CMAJ. 2010;182(6): 551-557.

15. Wang $\mathrm{H}$, Robinson RD, Johnson $\mathrm{C}$, et al. Using the LACE index to predict hospital readmissions in congestive heart failure patients. $B M C$ Cardiovasc Disord. 2014;14:97.

16. Bashir B, Schneider D, Naglak MC, Churilla TM, Adelsberger M. Evaluation of prediction strategy and care coordination for COPD readmissions. Hosp Pract (1995). 2016;44(3):123-128.

17. Quan H, Li B, Couris CM, et al. Updating and validating the Charlson Comorbidity Index and score for risk adjustment in hospital discharge abstracts using data from 6 countries. Am J Epidemiol. 2011;173(6):676-682.

18. Tan SY, Low LL, Yang Y, Lee KH. Applicability of a previously validated readmission predictive index in medical patients in Singapore: a retrospective study. BMC Health Serv Res. 2013;13:366. 
19. Amalakuhan B, Kiljanek L, Parvathaneni A, Hester M, Cheriyath P, Fischman D. A prediction model for COPD readmissions: catching up, catching our breath, and improving a national problem. J Community Hosp Intern Med Perspect. 2012;2:1.

20. Sharif R, Parekh TM, Pierson KS, Kuo YF, Sharma G. Predictors of early readmission among patients 40 to 64 years of age hospitalized for chronic obstructive pulmonary disease. Ann Am Thorac Soc. 2014;11(5):685-694.

21. Cotter PE, Bhalla VK, Wallis SJ, Biram RW. Predicting readmissions poor performance of the LACE index in an older UK population. Age Ageing. 2012;41(6):784-789.

22. Gruneir A, Dhalla IA, van Walraven C, et al. Unplanned readmissions after hospital discharge among patients identified as being at high risk for readmission using a validated predictive algorithm. Open Med. 2011;5(2):e104-e111.

23. Echevarria C, Steer J, Heslop-Marshall K, et al. The PEARL score predicts 90-day readmission or death after hospitalisation for acute exacerbation of COPD. Thorax. 2017;72(8):686-693.

24. McCullough PA, Hollander JE, Nowak RM, et al. Uncovering heart failure in patients with a history of pulmonary disease: rationale for the early use of B-type natriuretic peptide in the emergency department. Acad Emerg Med. 2003;10(3):198-204.

25. Hawkins NM, Petrie MC, Jhund PS, Chalmers GW, Dunn FG, McMurray JJV. Heart failure and chronic obstructive pulmonary disease: diagnostic pitfalls and epidemiology. Eur J Heart Fail. 2009;11(2): 130-139.

26. Coventry PA, Gemmell I, Todd CJ. Psychosocial risk factors for hospital readmission in COPD patients on early discharge services: a cohort study. BMC Pulm Med. 2011;11:49.
27. Carey K, Lin M-Y. Hospital length of stay and readmission: an early investigation. Med Care Res Rev. 2014;71(1):99-111.

28. Hurst JR, Donaldson GC, Quint JK, Goldring JJ, Baghai-Ravary R, Wedzicha JA. Temporal clustering of exacerbations in chronic obstructive pulmonary disease. Am J Respir Crit Care Med. 2009;179(5):369-374.

29. Shah T, Churpek MM, Coca Perraillon M, Konetzka RT. Understanding why patients with COPD get readmitted: a large national study to delineate the Medicare population for the readmissions penalty expansion. Chest. 2015;147(5):1219-1226.

30. Benbassat J, Taragin M. Hospital readmissions as a measure of quality of health care: advantages and limitations. Arch Intern Med. 2000;160(8):1074-1081.

31. van Walraven C, Bennett C, Jennings A, Austin PC, Forster AJ. Proportion of hospital readmissions deemed avoidable: a systematic review. CMAJ. 2011;183(7):E391-E402.

32. Parikh R, Shah TG, Tandon R. COPD exacerbation care bundle improves standard of care, length of stay, and readmission rates. Int J Chron Obstruct Pulmon Dis. 2016;11:577-583.

33. Zafar MA, Panos RJ, Ko J, et al. Reliable adherence to a COPD care bundle mitigates system-level failures and reduces COPD readmissions: a system redesign using improvement science. BMJ Qual Saf. 2017;26(11):908-918

34. Bhatt SP, Wells JM, Iyer AS, et al. Results of a Medicare bundled payments for care improvement initiative for chronic obstructive pulmonary disease readmissions. Ann Am Thorac Soc. 2017;14(5):643-648.

35. Brotman DJ, Hoyer EH, Leung C, Lepley D, Deutschendorf A. Associations between hospital-wide readmission rates and mortality measures at the hospital level: are hospital-wide readmissions a measure of quality? J Hosp Med. 2016;11(9):650-651.
Clinical Epidemiology

\section{Publish your work in this journal}

Clinical Epidemiology is an international, peer-reviewed, open access, online journal focusing on disease and drug epidemiology, identification of risk factors and screening procedures to develop optimal preventative initiatives and programs. Specific topics include: diagnosis, prognosis, treatment, screening, prevention, risk factor modification,

Submit your manuscript here: https://www.dovepress.com/clinical-epidemiology-journal

\section{Dovepress}

systematic reviews, risk and safety of medical interventions, epidemiology and biostatistical methods, and evaluation of guidelines, translational medicine, health policies and economic evaluations. The manuscript management system is completely online and includes a very quick and fair peer-review system, which is all easy to use. 